\title{
A national survey of Canadian ophthalmologists to determine awareness of published guidelines for the management of uveitis
}

\author{
Crystal S. Y. Cheung ${ }^{1,4^{*}}$, Nima Noordeh ${ }^{2}$ and Chloe C. Gottlieb 2,3
}

\begin{abstract}
Background: The objectives of this study are to assess Canadian ophthalmologists' awareness of established uveitis treatment guidelines and clinical management of uveitis and to assess the frequency of government applications for immunomodulatory therapy (IMT) and identify primary prescribers.

A 25-item questionnaire was sent to 759 practicing Canadian ophthalmologists. Six questions assessed demographics including the year of residency completion, training by uveitis specialists during residency, and fellowship training. Five questions assessed application of guidelines to clinical scenarios, and 12 questions assessed referral patterns and success of obtaining coverage for IMT.

Results: Of 144 respondents, 12 (8.3\%) were uveitis specialists; $45.1 \%$ of respondents had uveitis training during residency by a uveitis specialist. Sixty-one percent reported awareness of management guidelines. Recent graduates (2001-2012) referred patients to uveitis specialists (55.3\%) less frequently than earlier graduates. Recent graduates also managed uveitis patients more frequently with corticosteroid injections (15.6\%) than those who graduated before 1980 (9.75\%). The majority (93.6\%) of respondents submitted less than six IMT funding applications for provincial drug coverage yearly, and $5.5 \%$ reported prescribing IMT themselves, rather than referring to other specialists.

Conclusions: Although greater than half of respondents reported awareness of uveitis treatment guidelines, Canadian ophthalmologists' awareness of uveitis treatment guidelines and application of the guidelines to patient care could be improved. Few applications are made for IMT, and the majority of applications are sent by non-ophthalmologists. This suggests the need for further education of ophthalmologist about uveitis treatment guidelines and for more ophthalmologists trained to manage uveitis with IMT.
\end{abstract}

Keywords: Uveitis, Guidelines, Management

\footnotetext{
* Correspondence: cheuncsy@gmail.com

This work was presented at the 2013 Association for Research in Vision and Ophthalmology Annual Meeting, Seattle, Washington, May 5-9, 2013 (poster presentation), and at the 31st Annual Meeting of the Canadian Ophthalmology Society, Montreal, Quebec, June 14-17, 2013 (paper presentation).

${ }^{1}$ Faculty of Medicine, University of Ottawa, Ottawa, Ontario, Canada

${ }^{4}$ Department of Ophthalmology, Kensington Eye Institute, 340 College Street,

Suite 600, Toronto, ON M5T 3A9, Canada

Full list of author information is available at the end of the article
} 


\section{Background}

Uveitis, a group of ocular inflammatory conditions, accounts for 10 to $15 \%$ of blindness in developed countries $[1,2]$. As per standard nomenclature, chronic uveitis is defined as persistent uveitis characterized by prompt relapse (in less than 3 months) after discontinuation of therapy [3]. Persistent uveitis is defined as inflammation greater than 3 months in duration [3]. Recurrent uveitis is repeated episodes of uveitis separated by periods of inactivity without treatment, in which these periods of inactivity without treatment are at least 3 months in duration [3]. Sight-threatening disease arises from chronic, persistent, or recurrent inflammation. Left untreated or undertreated, chronic, persistent, or recurrent inflammation may lead to complications, such as cataract, glaucoma, macular edema, retinal or choroidal inflammation and atrophy, intraocular hemorrhage, or optic nerve atrophy. The therapeutic goal is to prevent vision loss from these complications by controlling or eliminating chronic, persistent, or recurrent inflammation. Systemic corticosteroid and locally administered corticosteroid injections treat ocular inflammation during acute episodes of uveitis, but the side effects of multiple pulse treatments or prolonged systemic corticosteroid administration limit their convenience as monotherapy for chronic and persistent uveitis, except in selected cases where dexamethasone or fluocinolone implants have been shown to be successful as monotherapy [4]. Persistent or chronic cases of uveitis, where corticosteroid therapy has failed to adequately treat disease, require long-term immunomodulatory therapy (IMT) to treat active inflammation and reduce the frequency of recurrences [5]. The uveitis management guidelines developed by an international panel of experts recommends the initiation of corticosteroid-sparing IMT if intraocular inflammation cannot be controlled with $<10 \mathrm{mg} /$ day of prednisone within 3 months [5]. However, in the recent survey of uveitis treatment patterns in the USA, Nguyen et al. showed that $75 \%$ of the physician cohort surveyed was not aware of the aforementioned management guidelines [6]. The survey also indicated that the average corticosteroid dose was $44 \mathrm{mg} /$ day and this dose was maintained for an average duration of 21 months, which is much higher than the recommended tapered doses of corticosteroids to $<10 \mathrm{mg} /$ day within 3 months [5]. The high doses of corticosteroid contributed to the significant adverse effects observed in the cohort of uveitis patients in the survey. Therefore, more widespread awareness of the uveitis treatment guidelines, leading to less reliance on corticosteroids, and appropriate timing of corticosteroid-sparing IMT would likely improve patient outcomes.

The purpose of this study is to assess, among Canadian ophthalmologists, the current awareness of published guidelines for the treatment of uveitis and to evaluate whether practice patterns are congruent with the published uveitis treatment guidelines. This study also assessed the frequency of applications to the government and private insurance providers for immunomodulatory drugs or biologics.

\section{Methods \\ Survey population and development}

The study was approved by the Ottawa Health Science Network Research Ethics Board. A 25-item questionnaire was developed by the authors and distributed electronically to 759 practicing Canadian ophthalmologists via the Canadian Ophthalmological Society (COS). Residents and fellows were not included in this study. Informed consent was implied with the completion and electronic submission of the survey. No remuneration was given to respondents. Data was collected over a 4-month period from August 2012 to November 2012.

The following data were collected: the year of residency completion, presence of a uveitis specialist at the respondent's center of residency training, fellowship training in uveitis, and other sub-specialities including pediatric ophthalmology, retina, anterior segment and others, and percentage of patients with uveitis in the respondent's practice. Practice pattern data was also collected. This included awareness and utilization of uveitis treatment guidelines, referral patterns and associated barriers to referrals, co-management of uveitis with uveitis specialists or non-ophthalmologists, number of IMT applications for provincial and private insurance drug coverage, and success of obtaining IMT from these providers. Understanding and application of the aforementioned uveitis treatment guidelines was assessed by survey questions comprising definitions and clinical scenarios (Additional file 1).

\section{Data analysis}

Demographic and other data were tabulated. The proportion of total correct responses to the clinical scenario questions was defined as the proportion of cumulative correct responses to each question. Non-responses were not included in the proportions. Descriptive analyses were conducted where appropriate, and confidence interval (CI) for proportions was calculated using the exact method.

Statistical analysis was performed using SAS software (SAS Institute Inc., Cary, NC). Analyses were considered to be significant if $p<0.05$; reported $p$ values were not adjusted for multiple testings and should be considered nominal. Use of Fisher's exact test and chi-squared test was based on sample sizes. Fisher's exact test was used to determine whether awareness of uveitis management guidelines differed according to the following training circumstances: (1) sub-specialty training, (2) fellowship 
training in uveitis, and (3) presence of a uveitis specialist at the respondents' center of residency training. Chisquared test was used in the comparison of the proportion of total correct responses to clinical scenario questions to the following variables: (1) sub-specialty training, (2) fellowship training in uveitis, and (3) presence of a uveitis specialist at the respondents' center of residency training.

The year of residency completion was categorized into three groups: (1) 1960 to 1980, (2) 1981 to 2000, and (3) 2001 to 2012. Multivariate analysis was used to determine whether the year of residency completion was associated with the proportion of total correct responses to clinical scenario questions. Descriptive analyses were used to compare the year of residency completion with the frequency of selecting "corticosteroid injections" as response to clinical scenario questions and frequency of referral to uveitis specialists.

\section{Results}

The response rate of the survey was $19.23 \%(146 / 759)$, and $87.7 \%(128 / 146)$ of respondents completed the survey in its entirety. Demographic and referral pattern data are detailed in Table 1. Among the respondents, $8.3 \%$ $(12 / 146 ; \mathrm{CI}=0.04-0.14)$ were uveitis specialists. A fellowshiptrained uveitis specialist was present at $45.8 \%(66 / 148$; $\mathrm{CI}=0.36-0.53)$ of the respondents' center of residency training. Overall, $93.8 \%(137 / 146 ; \mathrm{CI}=0.89-0.97)$ of the physicians' practices consisted of less than $30 \%$ of uveitis patients. The majority of respondents $(73.3 \%$, $107 / 146 ; C I=0.65-0.80)$ indicated they have referred patients to uveitis specialists.

Self-reported awareness of the uveitis management guidelines was $61.0 \%(89 / 146 ; \mathrm{CI}=0.53-0.69)$, and $51.7 \%$ (74/143; 3 no responses; $\mathrm{CI}=0.43-0.60)$ of respondents indicated the utilization of these guidelines. The proportion of total correct responses to the clinical scenario questions was not statistically different $(p=0.547)$ between physicians who reported awareness of the aforementioned guidelines and those who did not. Awareness of guidelines did not differ significantly between respondents who were trained in uveitis $(p=0.127)$, respondents with sub-speciality training $(p=0.179)$, and presence of a fellowship-trained uveitis specialist at the respondent's center of residency training $(p=0.232)$ (Table 2).

Adherence to the uveitis management guideline recommendations [1] was evaluated using clinical scenario questions. The association between adherence to guidelines and different training circumstances was assessed. A statistically significant difference in the proportion of total correct responses to clinical scenario questions was also found between those with fellowship training in sub-specialities and those with no fellowship training $(p=0.0145)$. However, the proportion of total correct responses to the clinical scenario questions was not
Table 1 Demographics and referral pattern data of respondents

\begin{tabular}{lll}
\hline Question & $n(\%)$ & $\mathrm{Cl}$ \\
\hline Type of clinical practice & & \\
Community practice & $91(63.2)$ & $0.55-0.71$ \\
Part-time academic & $21(14.6)$ & $0.09-0.21$ \\
Full-time academic & $32(22.2)$ & $0.16-0.30$ \\
No response & 2 & \\
Year of ophthalmology residency completion & & \\
1960-1970 & $7(4.8)$ & $0.02-0.09$ \\
1971-1980 & $23(15.8)$ & $0.10-0.23$ \\
1981-1990 & $43(29.5)$ & $0.22-0.37$ \\
1991-2000 & $34(23.3)$ & $0.17-0.31$ \\
2001-2012 & $39(26.7)$ & $0.18-0.35$ \\
No response & 0 & \\
Sub-specialty or fellowship training & & \\
No fellowship training & $67(45.9)$ & $0.35-0.51$ \\
Retina & $24(16.4)$ & $0.10-0.22$ \\
Pediatrics & $11(7.5)$ & $0.04-0.12$ \\
Uveitis & $12(8.2)$ & $0.04-0.13$ \\
Cornea/anterior segment & $15(10.3)$ & $0.05-0.15$ \\
Other & $27(18.5)$ & $0.12-0.24$ \\
No response & 0 &
\end{tabular}

Fellowship-trained uveitis specialist present at the respondents' center of residency training

$\begin{array}{lll}\text { Yes } & 66(45.8) & 0.37-0.54 \\ \text { No } & 78(54.2) & 0.46-0.62 \\ \text { No response } & 2(1.37) & \end{array}$

Percentage of patients with uveitis in respondents' clinical practice

$\begin{array}{lll}>60 \% & 2(1.4) & 0.002-0.05 \\ 30-60 \% & 7(4.8) & 0.02-0.09 \\ <30 \% & 137(93.8) & 0.89-0.97 \\ \text { No response } & 0 & \end{array}$

Referral to uveitis specialists

$\begin{array}{lll}\text { Yes } & 107(73.3) & 0.65-0.80\end{array}$

$\begin{array}{lll}\text { No } & 27(18.5) & 0.13-0.26\end{array}$

Respondent is a uveitis specialist $\quad 12(8.2) \quad 0.04-0.14$

No response 0

Barriers of referral encountered when referring patients to uveitis specialist

\begin{tabular}{lll} 
Geography (distance) & $38(32.2)$ & $0.19-0.34$ \\
Wait time & $46(39.0)$ & $0.24-0.39$ \\
None & $40(33.9)$ & $0.20-0.35$ \\
Not application. Care for patients with uveitis & $15(12.7)$ & $0.06-0.16$ \\
Other & $8(6.78)$ & $0.02-0.10$ \\
No response & 28 & \\
\hline
\end{tabular}

$n$ number of respondents 
Table 2 Awareness of uveitis management guidelines in association with different training circumstances

\begin{tabular}{|c|c|c|c|c|}
\hline Awareness of treatment guidelines & Aware, $n(\%)$ & Not aware, $n(\%)$ & $p$ value & $\mathrm{Cl}$ \\
\hline $\begin{array}{l}\text { Stratified by the presence of a fellowship-trained uveitis } \\
\text { specialist at the center of residency training }\end{array}$ & $89(50.0 \%)$ & $57(39.3)$ & 0.232 & -0.057 to 0.26 \\
\hline Stratified by fellowship training in uveitis & $10(83.30 \%)$ & $2(16.70 \%)$ & 0.127 & $N A, n<5$ \\
\hline Stratified by fellowship training in a related sub-specialty & $50(64.96 \%)$ & $27(35.06 \%)$ & 0.179 & -0.05 to 0.28 \\
\hline
\end{tabular}

$n$ number of respondents, $\mathrm{Cl}$ confidence interval, NA not applicable

statistically significant across the following groups of comparisons: whether the respondent had fellowship training in uveitis $(p=0.0509)$ and whether a uveitis specialist was present at the respondent's center of residency $(p=0.698)$ (Table 3$)$.

The year of residency completion was categorized into three groups: 1960 to 1980,1981 to 2000, and 2001 to 2012. The proportion of total correct responses to clinical scenario questions in association with the year of residency completion is detailed in Table 3. When comparing the proportion of total correct responses in clinical scenario questions to the year of residency completion, there was a statistically significant difference between respondents who completed residency during 2000 to 2012 and those during 1960 to 1981 ( $p=0.0208)$ and 1981 to 2000 $(p=0.0067)$. The selection of corticosteroid injections (intravitreal or periocular) in the clinical scenario questions, which was an incorrect response, was $7.1 \%$ among graduates from 1960 to 1980, $12.4 \%$ in graduates from 1981 to 2000 , and $15.6 \%$ in graduates from 2001 to 2012. Referral to a uveitis specialist was $90.0 \%$ (27/30; CI $=0.73-0.98)$ among those who completed residency during 1960 to $1980,76.6 \%(59 / 77 ; \mathrm{CI}=$ $0.63-0.83)$ for graduates in 1981 to 2000 , and $53.8 \%$ (21/39; CI = 0.37-0.70) in 2001 to 2012 (Fig. 1).

The frequency of IMT applications and success of obtaining IMT are shown in Table 4. When IMTs are required, $5.5 \%$ of physicians $(8 / 145 ; 1$ no response; $\mathrm{CI}=$ 0.02-0.11) would prescribe the IMT themselves, while $54.5 \%(79 / 145 ; \mathrm{CI}=0.46-0.63)$ would seek prescription of the IMT from a rheumatologist, dermatologist, or general internist, and $40.0 \%(58 / 145 ; \mathrm{CI}=0.32-0.48)$ would refer to an ophthalmologist to coordinate the acquisition of IMT. Overall, $93.6 \%$ (117/125; 21 no responses; $\mathrm{CI}=0.88-0.97)$ of physicians make less than six IMT applications per year, and $89.0 \%$ (105/118; 28 no responses) of respondents make less than six co-IMT applications with rheumatologists or other physicians. Less than $10 \%$ of IMT applications was successfully obtained through private insurance and provincial drug coverage among $64.0 \%$ of physicians (71/111; 35 no responses; $\mathrm{CI}=0.54-0.73)$ and $56.5 \%$ of physicians $(61 / 108$; 38 no responses; $\mathrm{CI}=0.47$ $0.66)$, respectively. The frequency of IMT applications was $25.0 \%(17 / 68 ; 78$ no responses; $\mathrm{CI}=0.15-0.37)$ for

Table 3 Total response rate to uveitis management questions in association with awareness of treatment guidelines, different training circumstances, and the year of residency completion

\begin{tabular}{|c|c|c|c|c|}
\hline Stratification & $\begin{array}{l}\text { Number of } \\
\text { responses, } N\end{array}$ & $\begin{array}{l}\text { Number of correct } \\
\text { responses, } n(\%)\end{array}$ & $95 \%$ Confidence interval & $p$ value \\
\hline \multicolumn{5}{|c|}{ Awareness of uveitis treatment guidelines } \\
\hline Aware & 406 & $242(59.61)$ & $0.55-0.64$ & 0.5471 \\
\hline Not aware & 243 & $129(53.09)$ & $0.47-0.59$ & \\
\hline \multicolumn{5}{|l|}{ Fellowship training } \\
\hline Fellowship training & 348 & $189(54.31)$ & $0.49-0.60$ & 0.0145 \\
\hline No fellowship training & 301 & $192(63.79)$ & $0.58-0.69$ & \\
\hline \multicolumn{5}{|l|}{ Uveitis fellowship training } \\
\hline Uveitis-trained & 56 & $26(46.43)$ & $0.33-0.60$ & 0.0509 \\
\hline No uveitis training & 593 & $355(59.87)$ & $0.56-0.64$ & \\
\hline \multicolumn{5}{|c|}{ Uveitis specialist present at the center of the respondents' residency training } \\
\hline Uveitis specialist present & 288 & $166(57.64)$ & $0.52-0.63$ & 0.6981 \\
\hline No uveitis specialist present & 355 & $210(59.15)$ & $0.54-0.64$ & \\
\hline \multicolumn{5}{|l|}{ Year of residency completion } \\
\hline 1960-1980 & 131 & $84(64.12)$ & $0.55-0.72$ & 1981-2000 vs 1960-1980: 0.3596 \\
\hline $1981-2000$ & 352 & $214(60.80)$ & $0.55-0.66$ & 2001-2012 vs 1981-2000: 0.0208 \\
\hline $2001-2012$ & 166 & $83(50)$ & $0.42-0.58$ & $2001-2012$ vs 1960-1980: 0.0067 \\
\hline
\end{tabular}




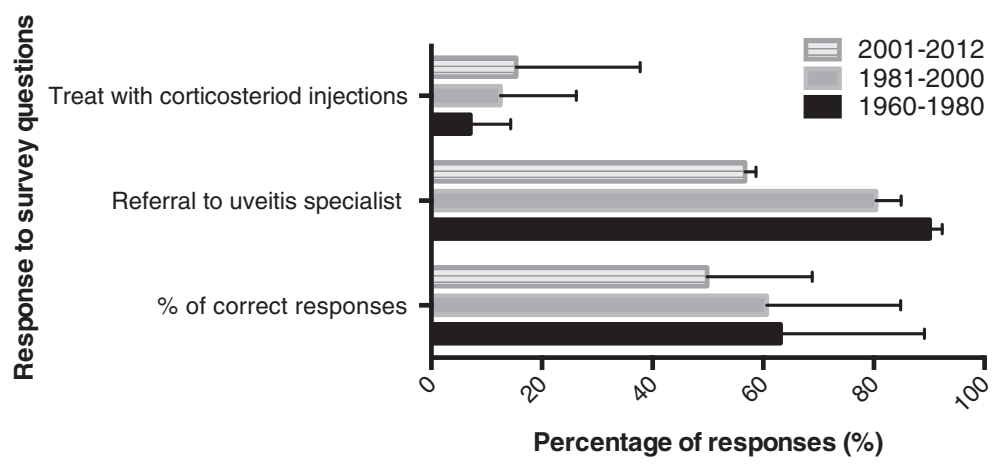

Fig. 1 The year of residency completion in association with the (a) proportion of total correct responses to clinical scenario questions ( $p=0.0208$ for 2001-2012 vs 1981-2000; $p=0.0067$ for 2001-2012 vs 1960-1980), (b) frequency of referral to uveitis specialists, and (c) frequency of managing patients with corticosteroid injections in clinical scenario questions

mycophenolate mofetil, $38.2 \%(26 / 68 ; \mathrm{CI}=0.27-0.51)$ for cyclosporine, $32.4 \%(22 / 68 ; \mathrm{CI}=0.22-0.45)$ for anti-tumor necrosis factor (TNF), and $36.8 \%(25 / 68$; $\mathrm{CI}=0.25-0.49)$ for others.

\section{Discussion}

Despite the well-recognized adverse effects of long-term corticosteroid usage, it has been the mainstay of uveitis management since its development [5, 7]. In addition to concerns about their adverse effects, high-dose systemic or locally injected corticosteroids, particularly serial periocular or intraocular corticosteroid injections, are not an optimal management strategy for chronic or frequently recurrent uveitis. At the time of writing, no Health Canadaapproved immunomodulatory drugs are indicated for uveitis and all such drugs are used off-label. A sustainedrelease dexamethasone intravitreal implant (Ozurdex; Allergan, Inc., Irvine, CA, USA) and Retisert (Bausch\&Lomb, Rochester, NY, USA) are currently the only Health Canadaapproved treatments for uveitis. However, these implants are only indicated for posterior uveitis and not anterior or intermediate uveitis [5]. Although studies have demonstrated the effectiveness of Ozurdex for non-infectious posterior uveitis, the protracted disease course of some patients with chronic uveitis, measured in years and decades, is lengthy compared to the 6-month duration of a steroid implant [8]. When treatment with systemic corticosteroid and/or multiple local injections or implants have failed to reduce intraocular inflammation to an acceptable level, then corticosteroid-sparing IMT is warranted. An international expert panel has published guidelines recommending the appropriate initiation of corticosteroid-sparing IMT. [5]

To the best our knowledge, this is the first study examining the awareness of and adherence to uveitis management guidelines among Canadian ophthalmologists. This study described the awareness and utilization of the aforementioned guidelines, practice pattern variations across different years of residency completion, and the frequency of prescribing IMT and co-management of uveitis patients.

\section{Awareness of and adherence to uveitis management guidelines}

In this survey, self-reported awareness of the uveitis management guidelines was $61.0 \%(\mathrm{CI}=0.53-0.69)$ and $51.7 \%(\mathrm{CI}=0.43-0.60)$ of respondents indicated utilization of these guidelines. The level of awareness of the aforementioned guidelines found in this study is higher than those found by Nguyen et al., where $25 \%$ of the US physician cohort surveyed was aware of the aforementioned management guidelines $[4,5]$. In addition, the results of this survey showed that self-reported awareness of guidelines was not associated with significantly higher proportion of total correct responses to the clinical scenario questions $(p=0.547)$, suggesting that ophthalmologists who reported awareness of the uveitis management guidelines did not use the guidelines in their clinical practice.

Factors which influence respondents' awareness of treatment guidelines were assessed. Awareness and unawareness of guidelines did not differ significantly for respondents who were trained in uveitis $(p=0.127)$, respondents with any sub-speciality training $(p=0.179)$, and presence of fellowship-trained uveitis speciality at the respondent's center of residency training $(p=0.232)$ (Table 2). These cumulative results suggest that awareness of published recommendations was not affected by sub-specialty training (including uveitis training) or residency training by a uveitis specialist. Despite the large difference observed between self-reported awareness (83.30\%; CI not applicable) and unawareness (16.70\%; CI not applicable) of management guidelines among the uveitis specialists, no statistically significant difference was found. This may be attributable to inadequate power of the analysis due to the small number of uveitis specialists (12) who responded to this survey. 
Table 4 Immunomodulatory therapy applications to public and private insurance providers

\begin{tabular}{|c|c|c|}
\hline Question & $n(\%)$ & $95 \% \mathrm{Cl}$ \\
\hline \multicolumn{3}{|l|}{ How do you obtain immunomodulatory drugs or biologics for your patients? } \\
\hline Request from a rheumatologist, dermatologist, gastroenterologist, or internist & $79(54.5)$ & $0.46-0.62$ \\
\hline Refer to an ophthalmologist who will prescribe or coordinate further care for patients with uveitis & $58(40.0)$ & $0.32-0.48$ \\
\hline Prescribe yourself & $8(5.5)$ & $0.02-0.11$ \\
\hline No response & 1 & \\
\hline \multicolumn{3}{|l|}{ Refer patients to a uveitis specialist } \\
\hline Yes & $107(73.3)$ & $0.65-0.80$ \\
\hline No & $27(18.5)$ & $0.13-0.26$ \\
\hline I am a uveitis specialist & $12(8.2)$ & $0.04-0.14$ \\
\hline No response & 0 & \\
\hline \multicolumn{3}{|l|}{ Number of new applications made to the government per year for IMT coverage } \\
\hline $0-5$ & $117(93.6)$ & $0.88-0.97$ \\
\hline $6-10$ & $5(4.0)$ & $0.01-0.09$ \\
\hline $11-20$ & $2(1.6)$ & $0.002-0.06$ \\
\hline $21-30$ & $1(0.8)$ & $0.0002-0.04$ \\
\hline$>30$ & 0 & \\
\hline No response & 21 & \\
\hline \multicolumn{3}{|l|}{ Number of co-applications for IMT made with rheumatologists or other physicians } \\
\hline $0-5$ & $105(89.0)$ & $0.69-0.84$ \\
\hline $6-10$ & $10(8.5)$ & $0.03-0.13$ \\
\hline $11-20$ & $2(1.7)$ & $0.002-0.05$ \\
\hline $21-30$ & 0 & $0.00-0.03$ \\
\hline$>30$ & $1(0.8)$ & $0.002-0.04$ \\
\hline No response & 28 & \\
\hline \multicolumn{3}{|c|}{ Percentage of respondent's patients with their IMT successfully covered through government or provincial health plan } \\
\hline$<10 \%$ & $71(64.0)$ & $0.54-0.73$ \\
\hline $10-50 \%$ & $32(28.8)$ & $0.21-0.38$ \\
\hline $51-90 \%$ & $5(4.5)$ & $0.02-0.10$ \\
\hline$>91 \%$ & $3(2.7)$ & $0.006-0.08$ \\
\hline No response & 35 & \\
\hline \multicolumn{3}{|l|}{ Percentage of respondent's patients with their IMT successfully covered through private insurance } \\
\hline$<10 \%$ & $61(56.5)$ & $0.46-0.66$ \\
\hline $10-50 \%$ & $29(26.9)$ & $0.19-0.36$ \\
\hline $51-90 \%$ & $10(9.3)$ & $0.04-0.16$ \\
\hline$>91 \%$ & $8(7.4)$ & $0.03-0.14$ \\
\hline No response & 38 & \\
\hline \multicolumn{3}{|l|}{ Class of IMT applied through the Exceptional Access Program } \\
\hline Mycophenolate mofetil & $17(25.0)$ & $0.11-0.28$ \\
\hline Anti-TNF & $22(32.4)$ & $0.16-0.35$ \\
\hline Cyclosporine & $26(38.2)$ & $0.20-0.39$ \\
\hline Others & $25(36.8)$ & $0.19-0.38$ \\
\hline No response & & \\
\hline
\end{tabular}

IMT immunomodulatory therapy, TNF tumor necrosis factor 
The associations between adherence to guidelines and different training circumstances were assessed. Adherence to guideline recommendations was determined by the proportion of total correct responses to clinical scenario questions. The proportion of total correct responses was significantly lower for respondents who had previous sub-specialty fellowship training than those who did not $(p=0.0145)$, suggesting that respondents with comprehensive ophthalmology practices were more likely to correctly identify cases where referral for IMT is necessary. Initially, this finding appears to contradict our hypothesis that additional fellowship training may be associated with higher likelihood of correctly identifying responses in clinical scenario questions. This apparent inconsistency may be due to the complexity of the cases presented in the clinical scenario questions, which focused on the management of chronic or recurrent uveitis. Multiple studies have shown that community-based comprehensive ophthalmologists manage a greater proportion of anterior uveitis and responsive uveitis cases than the ophthalmologists in university referral centers [9-11]. Uveitis cases which are refractory to treatment or chronic are more frequently managed by university-based ophthalmologists, most frequently uveitis specialists [9-11]. Since communitybased ophthalmologists are more likely to refer treatmentrefractory patients to specialists, this may explain the greater proportion of total correct responses observed in this study.

In contrast, the proportion of total correct responses was not significantly different between uveitis specialists and those without uveitis fellowship training ( $p=0.0509)$. This analysis may not be accurate due to the limited number of uveitis specialists included in this study, and as such, the results are inconclusive. The proportion of total correct responses was also not significantly higher between respondents who had a uveitis specialist present at the academic center where they completed residency training and those who did not $(p=0.6981)$. As such, ophthalmology residents may have limited interactions with these specialists.

\section{Uveitis management patterns among the most recent graduates}

The year of residency completion was associated with a lower proportion of total correct responses. In particular, the most recent graduates (i.e., completed residency between 2001 and 2012) were less likely to correctly answer the clinical scenario questions compared to respondents who completed residency from 1960 to $1980(p=0.0067)$ and from 1981 to $2000(p=0.0208)$. This suggests the most recent graduates were less likely to correctly identify uveitis cases where IMT induction is necessary based on the management guidelines [5]. In the 2008 Canadian survey evaluating residents and recent graduates' perception of competencies in different areas of clinical training, the sub-specialties where most respondents reported insufficient exposure were low-vision rehabilitation (77.5\%), refraction and glasses prescription (65\%), and neuroophthalmology (45\%); only approximately $15 \%$ of respondents indicated that clinical exposure to uveitis was inadequate in achieving competency [12]. The study by Zhou et al. described "adequate clinical exposure" broadly and did not specify exposures to investigations, diagnosis, or management strategies [12]. In contrast, the clinical scenario question in this survey focused on uveitis management, particularly the appropriate initiation of corticosteroid-sparing IMT and appropriate timing of referral to a uveitis specialist for chronic uveitis management. Nonetheless, the discrepancy between recent graduates' perception of competence in uveitis and their lower proportion of correct responses to clinical scenario questions suggests the importance of raising awareness of uveitis management guidelines in Canadian residency programs.

Self-reported referral rate to a uveitis specialist was the lowest among the most recent graduates (53.8\%; CI = 0.37-0.70) compared to those who completed residency during 1960 and 1980 (90.0\%; CI = 0.73-0.98) and during 1981 to 2000 (76.6\%; CI =0.66-0.85). The lower rate of referrals among newer graduates found in this survey parallels the results of the 2004 Canadian National Uveitis Survey [13]. In addition to differences in the proportion of referrals, recent graduates were also more likely to manage IMT-requiring uveitis with off-label corticosteroid injections. This observation may be attributable to the rapid increase of intravitreal injections performed for posterior segment diseases in the past decade $[14,15]$. In this case, the increased likelihood to treat uveitis with corticosteroid injections among the most recent graduates may contribute to the lower proportion of referrals observed.

\section{Applications for immunomodulatory therapy coverage to provincial drug coverage and private providers}

Immunomodulatory agents are indicated for corticosteroidsparing in patients with recurrent or chronic ocular inflammation where the dose of systemic corticosteroid is $\geq 10 \mathrm{mg} /$ day or $\geq 0.1 \mathrm{mg} / \mathrm{kg} /$ day and duration of corticosteroid therapy is $\geq 3$ months $[5,16]$. The adverse effects of corticosteroid therapy have been shown to be more frequent than IMT $[17,18]$. However, many ophthalmologists do not have extensive training in IMT administration, and monitoring and collaboration with uveitis specialists, rheumatologists, or internists is necessary [4]. Our results showed that only $5.5 \%(\mathrm{CI}=$ 0.02-0.11) of physicians would prescribe IMT themselves and $93.6 \%(\mathrm{CI}=0.88-0.97)$ make less than six IMT applications for provincial and private provider drug coverage per year. Thus, IMT initiation by ophthalmologists is 
infrequent. When IMT is required, $54.5 \%(\mathrm{CI}=0.46-$ 0.63 ) of respondents would coordinate IMT acquisition with specialists such as rheumatologists, dermatologists, or general internists. However, $89.0 \%(\mathrm{CI}=0.82-0.94)$ of respondents make less than six co-applications for IMT with rheumatologists or other physicians. Cumulatively, these results suggest that ophthalmologists make few IMT applications themselves and co-application with other physicians and majority of IMT applications are sent by non-ophthalmologists.

Over $73 \%(\mathrm{CI}=0.65-0.80)$ of respondents stated that they refer patients to uveitis specialists. When IMT initiation is required, $40.0 \%(\mathrm{CI}=0.32-0.48)$ of physicians would refer patients to uveitis specialists. Although majority of respondents refer patients to uveitis specialists, they also frequently identified geographic distance $(32.2 \%$; $\mathrm{CI}=0.24-0.41))$ and wait time $(39.0 \%$; $\mathrm{CI}=$ $0.30-0.48)$ as barriers. These barriers to referral may delay the initiation of IMT and result in further vision loss secondary to persistent ocular inflammation. Given the limitations of timely access to uveitis specialists, coordinating the acquisition of IMT with other specialists such as rheumatologists and internists is essential. Yet, the low number of co-IMT applications (fewer than six) made by ophthalmologists with other physicians is concerning.

The uveitis management guidelines outline the circumstances in which IMT induction is necessary [5]. However, there is limited information available on which IMT is considered first-line corticosteroid-sparing agents. Preferences for different IMT classes were assessed in this study. The results showed that the frequency of IMT applications was $25.0 \%(\mathrm{CI}=0.155-0.37)$ for mycophenolate mofetil, $32.4 \%(\mathrm{CI}=0.22-0.45)$ for anti-TNF, $38.2 \%(\mathrm{CI}=0.27-$ $0.51)$ for cyclosporine, and $36.8 \%(\mathrm{CI}=0.25-0.49)$ for others. This shows that the proportion of IMT drugs prescribed by ophthalmologists is similar across different types of IMT. In contrast to our findings, a survey of US uveitis specialists showed that methotrexate was preferred for initial management of uveitis while mycophenolate mofetil was preferred for intermediate and posterior uveitis; the primary reason for not prescribing cyclosporine was safety and tolerability. Another practice pattern survey of US uveitis specialists, Canadian specialists use cyclosporine more frequently, while mycophenolate mofetil was used less often [19].

\section{Limitations}

Several limitations of this study should be noted. First, the survey was set up such that mandatory responses to questions were not required and, therefore, incomplete data was recorded in the form of non-responses, resulting in incomplete data analysis and interpretation. A second limitation of this study is sampling bias, where physicians who responded to the study may feel more proficient in the management of uveitis leading to an overestimation of the proportion of self-reported awareness of guidelines and proportion of total correct responses. Therefore, the management patterns of uveitis among Canadian ophthalmologists may not be accurately represented through this survey. Third, the low number of uveitis specialists included in this study limits the statistical analyses. Fourth, there were many non-responses to questions regarding IMT applications to provincial and private drug coverage and this might have been due to respondents not making any IMT applications and the survey questions did not assess this directly. Lastly, it has been reported that indicators for treatment vary among uveitis specialists despite guidelines, and this variation is even greater among non-uveitis specialists. As such, this may overestimate the observed variability in responses to the survey [20].

\section{Conclusions}

In conclusion, the awareness and utilization of the uveitis management guidelines among Canadian ophthalmologists can be improved. Furthermore, recent graduates were less likely to identify cases of uveitis where treatment with IMT is indicated. They were also more likely to manage uveitis with corticosteroid injections and less likely to refer patients to uveitis specialists. When use of IMT is necessary, the majority of ophthalmologists indicated that they would refer patients to uveitis specialists or other internists such as rheumatologists and dermatologists. Few applications for IMT were made by ophthalmologists, and majority of these applications were sent by non-ophthalmologists. Cumulatively, these results suggest the need for further education of Canadian ophthalmologists regarding uveitis treatment guidelines and the need to increase the number of uveitis specialists in Canada.

\section{Additional file}

Additional file 1: Clinical scenarios in the uveitis survey. (doc $13 \mathrm{~kb}$ )

\section{Acknowledgements}

We thank Ms. Tinghua Zhang (MSc, affiliated with Ottawa Hospital Research Institute) for the statistical analysis. We also thank COS for the electronic distribution of our survey.

\section{Funding}

This study received financial support from Allergan Inc (unrestricted educational grant, Dr. Chloe Gottlieb.)

\section{Authors' contributions}

CG conceived the study. CC, NN and CG paricipated in its design, cooridnation and data collection. CC and CG were involved in the statisitcal analysis. CC, NN and CG were involved in the preparation of the manuscript. All authors read and approved in final manuscript.

Competing interests

The authors declare that they have no competing interests. 


\section{Ethics approval and consent to participate}

The study was approved by the Ottawa Health Science Network Research Ethics Board. Informed consent was implied with the completion and electronic submission of the survey.

\section{Author details}

'Faculty of Medicine, University of Ottawa, Ottawa, Ontario, Canada. ${ }^{2} U$ niversity of Ottawa Eye Institute, Ottawa, Ontario, Canada. ${ }^{3}$ Ottawa Hospital Research Institute, Ottawa, Ontario, Canada. ${ }^{4}$ Department of Ophthalmology, Kensington Eye Institute, 340 College Street, Suite 600, Toronto, ON M5T 3A9, Canada.

Received: 23 June 2016 Accepted: 22 August 2016

Published online: 18 October 2016

\section{References}

1. Durrani OM, Tehrani NN, Marr JE et al (2004) Degree, duration, and causes of visual loss in uveitis. Br J Ophthalmol 88(9):1159-1162

2. Jabs DA, Nussenblatt RB, Rosenbaum JT (2005) Standardization of uveitis nomenclature for reporting clinical data. Results of the First International Workshop. Am J Ophthalmol 140(3):509-516

3. Nussenblatt RB (1990) The natural history of uveitis. Int Ophthalmol 14:303-308

4. Durrani K, Zakka FR, Ahmed M et al (2011) Systemic therapy with conventional and novel immunomodulatory agents for ocular inflammation disease. Surv Ophthalmol 56:474-510

5. Jabs DA, Rosenbaum JT, Foster CS et al (2000) Guidelines for the use of immunosuppressive drugs in patients with ocular inflammatory disorders: recommendations of an expert panel. Am J Ophthalmol 130:492-513

6. Nguyen QD, Hatef E, Kayen B et al (2011) A cross-sectional study of the current treatment patterns in non-infectious uveitis among specialists in the United States. Ophthalmology 118:184-190

7. Kaufman HE (1964) Ocular inflammatory disease. N Engl J Med 270:456-463

8. Lowder C, Belfort R, Lightman $S$ et al (2011) Dexamethasone intravitreal implant for non-infectious intermediate or posterior uveitis. Arch Ophthalmol 129:545-553

9. McCannel CA, Holland GN, Helm CJ et al (1996) Causes of uveitis in the general practice of ophthalmology. UCLA Community-Based Uveitis Study Group. Am J Ophthalmol 121:35-46

10. Chan SM, Hudson M, Weis E (2007) Anterior and intermediate uveitis cases referred to a tertiary centre in Alberta. Can J Ophthalmol 42:860-864

11. Sakai IL, Usui Y, Sakai M et al (2010) Clinical statistics of endogenous uveitis: comparison between general eye clinic and university hospital. Int Ophthalmol 30:297-301

12. Zhou AW, Noble J, Lam WC (2009) Canadian ophthalmology residency training: an evaluation of resident satisfaction and comparison with international standards. Can J Ophthalmol 44:540-547

13. Forooghian F, Gupta R, Wong D et al (2006) Anterior uveitis investigation by Canadian ophthalmologists: insights from the Canadian National Uveitis Survey. Can J Ophthalmol 41:576-583

14. Peyman GA, Lad EM, Moshfeghi DM (2009) Intravitreal injection of therapeutic agents. Retina 29:875-912

15. Haller JA, Bandello F, Belfort R Jr et al (2010) Randomized, sham-controlled trial of dexamethasone intravitreal implant in patients with macular edema due to retinal vein occlusion. Ophthalmology 117:1134.e3-1146.e3

16. Cunningham ET Jr, Wender JD (2010) Practical approach to the use of corticosteroids in patients with uveitis. Can J Ophthalmol 45:352-358

17. Gregory AC II, Kempen JH, Daniel E et al (2013) Risk factors for loss of visual acuity among patients with uveitis associated with juvenile idiopathic arthritis: the Systemic Immunosuppressive Therapy for Eye Disease Study. Ophthalmology 120:186-192

18. Samson CM, Waheed M, Baltatzis S et al (2001) Methotrexate therapy for chronic noninfectious uveitis. Ophthalmology 108:1134-1139

19. Esterberg E, Acharya NR (2012) Corticosteroid-sparing therapy: practice patterns among uveitis specialists. J Ophthalmic Inflamm Infect 2:21-28

20. Cunningham ET Jr (2012) Surveying uveitis specialists - a call for consensus. J Ophthalmic Inflamm Infect 2:61-63

\section{Submit your manuscript to a SpringerOpen ${ }^{\circ}$ journal and benefit from:}

- Convenient online submission

- Rigorous peer review

Immediate publication on acceptance

- Open access: articles freely available online

- High visibility within the field

- Retaining the copyright to your article

Submit your next manuscript at $\boldsymbol{s p r i n g e r o p e n . c o m ~}$ 